\title{
LACTOSE AND D-GALACTOSE CATABOLISM IN THE FILAMENTOUS FUNGUS ASPERGILLUS NIDULANS
}

\author{
ERZSÉBet FeKete, JÁnOs PADRA, AtTILA SZENTIRMAi AND LEVENTE
}

KARAFFA*

\author{
${ }^{1}$ Department of Genetics and Applied Microbiology, Faculty of Science and Technology, \\ University of Debrecen, H-4010, P.O.Box 56., Debrecen, Hungary \\ *corresponding author. Tel.: +36 52512900 ext. 22488; Fax: +36 52533 677; \\ E-mail: lkaraffa@delfin.unideb.hu
}

\section{Introduction}

The disaccharide lactose (1,4-0-ß-D-galactopyranosyl-D-glucose) is a byproduct of cheese production accumulating to amounts of 800000 tons per year worldwide, of which 15 $\%$ is used as a carbon source for various microbial fermentations, such as cellulase production by Trichoderma reesei or penicillin production by Penicillium chrysogenum [1]. At present, lactose is the only derepressing carbon source used on industrial scale. Nevertheless, little is known about the regulation of its metabolism in filamentous fungi. Lactose is metabolized slowly, and some important fungi such as A. niger cannot use it at all. A more detailed knowledge on the rate-limiting steps would be helpful to improve its industrial application.

We have chosen A. nidulans as an object for investigating how lactose and galactose metabolism are regulated because it has long become a model system for biochemical and genetic research on fungi, and mutants in the lactose-metabolizing pathway of A. nidulans are available [2]. 


\section{General scheme of lactose and D-galactose catabolism in A. nidulans}

The first step in lactose metabolism is its transport by a lactose permease, followed by a hydrolysis to glucose and galactose by an intracellular ß-galactosidase [3]. D-glucose catabolism proceeds via either the glycolytic or the oxidative pentose phosphate pathway, while D-Galactose degradation was known to occur exclusively via the Leloir-pathway. The Leloir pathway is a ubiquitous trait in pro- and eukaryotic cells [4]. It can be used as a catabolic pathway for the degradation of D-galactose as an energy and carbon source while it links as an anabolic pathway the metabolism of carbohydrates, such as the synthesis of lipopolysaccharides, of cell wall components, and of exopolysaccharides for which galactosides are frequently required as building blocks. In cucumber, Leloir pathway was reported to be involved in the sucrose synthesis from stachyose [5]. It involves an ATPdependent galactokinase (EC 2.7.1.6) to form D-galactose 1-phosphate, which is subsequently transferred to UDP-glucose in exchange with D-glucose 1-phosphate by D-galactose 1phosphate-uridyltransferase (EC 2.7.7.12). The resulting UDP-galactose is a substrate for the reaction catalyzed by UDP-galactose 4-epimerase (EC 5.1.3.2), resulting in UDP-glucose.

\section{Regulation of formation of the ß-galactosidase activity}

Wild-type A. nidulans do not exhibit any ß-galactosidase activity on glucose or glycerol, while it is clearly present in mycelia grown on lactose. No extracellular or cellbound ß-galactosidase activity can be detected. The time-profile of activity essentially parallel growth and lactose consumption, and decline after exhaustion of the carbon source. The ßgalactosidase activity of mycelia growing on lactose can further raised by addition of galactose, indicating that enzyme formation is not maximal during growth on lactose $[3,6]$. 
The formation of $ß$-galactosidase on lactose or D-galactose, but not on glucose or glycerol suggests that its biosynthesis may be under carbon catabolite control. Addition of glucose to cultures growing on lactose indeed immediately decreases ß-galactosidase activity. Uptake of lactose is also suspended as long as glucose is present in the medium. After the consumption of glucose, lactose is consumed again and also ß-galactosidase activity is reinitiated.

These results demonstrate an immediate inhibition of the activity of ß-galactosidase by glucose, but do not distinguish between inducer exclusion and true carbon catabolite repression. Employing the $A$. nidulans mutant strain $\Delta 4$, in which the creA locus had been deleted [7], both lactose consumption and ß-galactosidase activity remain unaffected upon glucose pulse, indicating that lactose uptake is not inhibited but repressed by glucose.

When $A$. nidulans mutant strain $\Delta 4$ is grown on glucose and glycerol, mycelia form a clear, albeit low ß-galactosidase activity, corresponding to appr. $18 \%$ of the levels observed on lactose. This indicates that part of the ß-galactosidase formation is indeed subject to CreAdependent carbon catabolite regulation, but it is the induction that accounts for the major part of the activity. In fact, ß-galactosidase is effectively induced by the addition of D-galactose. Simultaneous addition of glucose and D-galactose do not inhibit the uptake of D-galactose, but reduced the $\beta$-galactosidase activity by about $30 \%$, thus indicating some effect of glucose also on the induction process. The involvement of CreA in this effect was again proven by the use of $A$. nidulans mutant strain $\Delta 4$, in which D-galactose induction of $ß$-galactosidase is completely unaffected.

Our data show that the effect of glucose occurs at least at three levels, which are all dependent on the carbon catabolite repressor CreA: (a) a blockade of constitutive ßgalactosidase formation; (b) a partial interference with induction, such that in a CreA-negative background $150 \%$ of the wild-type levels of induction were obtained; and (c) a repression of 
lactose uptake by glucose. Interference of CreA-dependent carbon catabolite regulation with gene expression at multiple regulatory levels have been reported in A. nidulans [7]. However, interference of CreA with inducer transporting permeases (such as here with the lactose permease) has not yet been shown.

\section{Reductive pathway of D-galactose utilization in A. nidulans}

Galactokinase is a key enzyme of the Leloir-pathway, and essential for the metabolism of D-galactose in yeast [8]. Consistent with this, an A. nidulans mutant in galactokinase (galE) was reported to be unable to grow on D-galactose $[9,10]$. In contrast, we have noted that A. nidulans galE mutants still grow on D-galactose [11]. A detailed comparison of the conditions revealed that the former authors used nitrate as a nitrogen source, whereas we used ammonium phosphate. Indeed, nitroge source strongly affects the ability of the galE mutant to grow on D-galactose: whereas the galE mutant exhibits a strongly reduced growth on Dgalactose with nitrate as a nitrogen source, it grows well with ammonium as a nitrogen source, independently of the anion of the ammonium salt (sulphate, chloride, phosphate). It was therefore concluded that there is an alternative pathway for D-galactose catabolism operating on media containing ammonium as a nitrogen source, which however is not operating in the presence of nitrate. Subsequent studies provided genetic and biochemical evidence for an alternative pathway for D-galactose degradation in A. nidulans, which involve reduction of Dgalactose to galactitol and oxidation of galactitol to L-sorbose, and for which we identified Larabinitol-4-dehydrogenase and hexokinase as essential enzymes.

It was long demonstrated that L-sorbose is not a carbon source for A. nidulans [9, 12]. However, evidence is now available that the wild-type and galE mutant strains are able to grow on L-sorbose when ammonium is used as a nitrogen source [11]. On the other hand, no growth is observed on a nitrate-containing medium with L-sorbose. At the moment, we are 
unable to provide scientific explanation to this, but speculate that L-sorbose catabolism similarly to the alternative reductive catabolism of D-galactose - is inhibited in the presence of nitrate.

The further fate of L-sorbose is still unclear. One possibility would be its catabolism via D-sorbitol and D-fructose [12] to D-fructose-6-phosphate. Alternatively, L-sorbose could be phosphorylated to L-sorbose-6-phosphate and subsequently isomerized to fructose-6phosphate. Both pathways may also operate simultaneously.

Irrespective of the steps catabolizing L-sorbose, the reductive D-galactose pathway is reminiscent of the fungal pathways for D-xylose or L-arabinose catabolism, which also proceed by reduction of the aldoses to the corresponding polyols, subsequent oxidation by polyol dehydrogenases to form a ketose, which then is phosphorylated [13]. The ketosephosphates are then isomerized to ribulose-5-phosphate, an intermediate of the pentose phosphate pathway and thus primary metabolism [14]. No such reactions are known however for hexoses, and we are therefore at present unaware whether a similar metabolic strategy will be implied here.

\section{Acknowledgement}

Research in our laboratory is supported by the Hungarian Scientific Research Fund (OTKA; F042602, D048617 and K67667) and the National Office for Research and Technology (NKTH; A2-2006-0017). Levente Karaffa is a recipient of a Bolyai János Research Scholarship (BO/00107/06) 


\section{References}

1. Roelfsema WA, Kuster FM, Pluim H: Lactose and derivatives. In: (Elvers B, Hawkins S, Schulz G, eds) Ullmann's Encyclopedia of Industrial Chemistry, $4^{\text {th }}$ ed. pp. $107-114$. VCH Weinheim FRG (1990).

2. Roberts CF: Enzyme lesions in galactose non-utilizing mutants of Aspergillus nidulans. Biochim. Biophys. Acta 201, 267 (1970).

3. Fekete E, Karaffa L, Sándor E, Seiboth B, Biró S, Szentirmai A, Kubicek CP: Regulation of formation of the intracellular $\beta$-galactosidase activity of Aspergillus nidulans. Arch. Microbiol. 179, 7 (2002).

4. Frey PA: The Leloir pathway: a mechanistic imperative for three enzymes to change the stereochemical configuration of a single carbon in galactose. FASEB J. 10, 461 (1996).

5. Gross KC, Pharr DM: A potential pathway for galactose metabolism in Cucumis sativus L., a stachyose transporting species. Plant Physiol. 69, 117 (1982).

6. Ilyés H, Fekete E, Karaffa L, Fekete É, Sándor E, Szentirmai A, Kubicek CP: CreAmediated carbon catabolite repression of ß-galactosidase formation in Aspergillus nidulans is growth rate dependent. FEMS Microbiol. Letts. 235, 147 (2004).

7. Dowzer CE, Kelly JM.: Analysis of the creA gene, a regulator of carbon catabolite repression in Aspergillus nidulans. Mol. Cell. Biol. 11, 5701 (1991).

8. Dickson RC, Riley MI: The lactose-galactose regulon of Kluyveromyces lactis. Bio/Technol. 13, 19 (1989).

9. Roberts CF: The genetic analysis of carbohydrate utilization in Aspergillus nidulans. J. Gen. Microbiol. 31, 45 (1963). 
10. Käfer E: Meiotic and mitotic recombination in Aspergillus and its chromosomal abberations. Adv. Genet. 19, 33 (1977).

11. Fekete E, Karaffa L, Sándor E, Bányai I, Seiboth B, Gyémánt G, Sepsi A, Szentirmai A, Kubicek CP: The alternative D-galactose degrading pathway of Aspergillus nidulans proceeds via L-sorbose. Arch. Microbiol. 18, 35 (2004).

12. Elorza MV, Arst HN Jr.: Sorbose resistant mutants of Aspergillus nidulans. Mol. Gen. Genet. 111, 185 (1971)

13. Witteveen CFB, Busink R, van der Vondervoort P, Dijkema C, Swart K, Visser J: Larabinose and D-xylose catabolism in Aspergillus niger. J. Gen. Microbiol. 135, 2163 (1989).

14. Shi NQ, Prahl K, Hendrick J, Cruz J, Lu P, Cho JY, Jones S, Jeffries T: Characterization and complementation of a Pichia stipitis mutant unable to grow on D-xylose or Larabinose. Appl. Biochem. Biotechnol. 84, 201 (2000). 